\title{
Removing Instrumental Artifacts: Suspension Violin Modes
}

\author{
Soma Mukherjee ${ }^{(1),(2)}$ and Lee Samuel Finn ${ }^{(1),(2),(3)}$ \\ (1) Center for Gravitational Physics and Geometry, The Pennsylvania State University; (2) \\ Department of Physics, The Pennsylvania State University; (3) Department of Astronomy and \\ Astrophysics, The Pennsylvania State University.
}

\begin{abstract}
We describe the design of a Kalman filter that identifies suspension violin modes in an interferometric gravitational wave detectors data channel. We demonstrate the filter's effectiveness by applying it to data taken on the LIGO 40M prototype.
\end{abstract}

\section{MOTIVATION}

The wire suspensions of the interferometer test masses are a conduit for environmental noise to enter the gravitational wave data channel; additionally, they are a source of noise themselves. Both the pendulum mode (whose frequency is out of the interferometers band) and the suspension wire violin modes (whose fundamental mode frequencies are in-band) are energized by their contact with the thermal bath. Owing to their weak damping this energy is strongly concentrated about the mode resonant frequencies. At the fundamental violin mode frequencies the thermal noise dominates the other noise sources by approximately $50 \mathrm{~dB}$. In addition to this thermal noise component, non-thermal excitations of the suspension wires (e.g., sudden creep events) can lead to excitations in the interferometer output.

These narrow band features are instrumental artifacts, not gravitational waves: along with other artifacts they should be removed from the data before it is studied for the presence of other signals. As instrumental artifacts, however, they carry important information about the instrument's state. Thermal and technical noise that disturbs the suspension excites these modes and move the mirrors, leading to an artifact in the gravitational wave channel. Gravitational waves give rise to a signal by changing the distance between the mirrors, but do not move or otherwise excite the suspension modes. Correspondingly, if we can determine the mode state as a function of time we have a way of eliminating a broad class of technical noise sources that might otherwise masquerade as gravitational wave bursts.

How can we identify the state of the suspension violin modes, given only the gravitational wave channel? This is a classic problem in data analysis, generally addressed by a Kalman filter. We have developed such a filter for use in LIGO and 
shown, using data taken in November 1994 at the LIGO 40M prototype, that it enables us to follow the state of the violin modes independently of the other noise sources - both technical and fundamental - that contribute to the gravitational wave channel.

\section{THE KALMAN FILTER}

The Kalman Filter [1] is a mechanism for predicting the multi-dimensional state of a dynamical system from a multi-dimensional observable. The system is assumed to evolve linearly and the observable is assumed to be linearly related to the state. denoting the system state $\mathbf{x}$ we have (for discrete time series):

$$
\mathbf{x}[k]=\mathbf{A} \cdot \mathbf{x}[k-1]+\mathbf{w}[k-1]
$$

We assume that the system is driven by a stochastic force, referred to as the process noise and denoted $\mathbf{w}$. The state dynamics determine the linear operator $\mathbf{A}$.

The state contributes to the observation $\mathbf{y}$, which also includes a stochastic, additive measurement noise $\mathbf{v}$ :

$$
\mathbf{y}[k]=\mathbf{C} \cdot \mathbf{x}[k]+\mathbf{v}[k]
$$

In the classic Kalman filter the process and measurement noises are assumed to be Normal processes with known co-variances $\mathbf{W}$ and $\mathbf{V} .^{1}$

Now suppose that we have an estimate $\widehat{\mathbf{x}}[k-1]$ of the state, and also an estimate of the error co-variance $\mathbf{P}[k-1]$ in the estimate, at sample $k-1$. The Kalman filter uses these estimates, the observation $\mathbf{y}[k]$ at sample $k$, and $\mathbf{A}, \mathbf{C}, \mathbf{W}$ and $\mathbf{V}$ to form an estimate of the state and its error co-variance at sample $k$ :

$$
\begin{aligned}
\widehat{\mathbf{x}}[k] & :=\mathbf{K}[k] \cdot(\mathbf{y}[k]-\widehat{\mathbf{y}}[k]) \\
\widehat{\mathbf{P}}[k] & :=(\mathbf{I}-\mathbf{K}[k] \cdot \mathbf{C}) \cdot \widetilde{\mathbf{P}}[k] \cdot(\mathbf{I}-\mathbf{K}[k] \cdot \mathbf{C})^{T}
\end{aligned}
$$

where

$$
\begin{aligned}
\widehat{\mathbf{y}}[k] & :=\mathbf{C} \cdot \mathbf{A} \cdot \widehat{\mathbf{x}}[k-1] \\
\mathbf{K}[k] & :=\widetilde{\mathbf{P}}[k] \cdot \mathbf{C}^{T} /\left(\mathbf{V}+\mathbf{C} \cdot \widetilde{\mathbf{P}}[k] \mathbf{C}^{T}\right) \\
\widetilde{\mathbf{P}}[k] & :=\mathbf{A} \cdot \widehat{\mathbf{P}}[k-1] \cdot \mathbf{A}^{T}+\mathbf{W} .
\end{aligned}
$$

The estimated system state $\mathbf{x}[k]$ (e.g., the generalized coordinate and conjugate momentum of the violin mode normal mode of the wire) is thus completely determined by the observation $\mathbf{y}[k]$, the estimated state at sample $k-1$, the wire dynamics,

1) Even when $\mathbf{w}$ and $\mathbf{v}$ are not Normal the Kalman filter estimates of the state $\mathbf{x}[k]$ can be shown to have the smallest mean-square error of all linear state estimators that depend only on the co-variances. 
and the statistical properties of the process and measurement noise. The error in the estimate $\mathbf{x}[k]$ falls with $k$, converging upon a limiting error covariance that is fully determined by $\mathbf{A}, \mathbf{C}, \mathbf{W}$ and $\mathbf{V}$; correspondingly, we can choose any initial estimate of $\mathbf{x}$ and $\mathbf{P}$ and the filter will, after several iterations, adjust the state estimate and error accordingly.

From the state estimate at each sample we can, through the measurement equation, estimate the contribution of the system to the actual observation. This estimated contribution can be subtractively removed from the actual observation, leaving a residual that is as free from the contaminating influence of the process as we can make it.

\section{MODELING THE VIOLIN MODES}

To describe a Kalman filter for our system we need a model for the wire state dynamics, the relationship between the wire state and the appearance of the mode in the detector data channel, and estimates of the process and measurement noise.

The wire motion contributes significantly to the data channel only in a narrow band about the violin mode resonant frequency; correspondingly, we can focus attention this narrow band and model the wire dynamics as a viscously damped harmonic oscillator driven by white noise ${ }^{2}$ :

$$
\ddot{\psi}=-\omega_{0}^{2} \psi-\gamma \dot{\psi}+N(t)
$$

We assume that the measurement is of the state variable $\psi$ plus white measurement noise.

There are many violin modes, corresponding to the many wires that are used to suspend the interferometer mirrors: for each wire there are separate state variables and a separate equation describing the dynamics of that mode.

Using standard lock-in techniques we mix the $\psi$ and the data channel with a local oscillator whose frequency is near that of the violin modes and band-limit the output of the lock-in to the narrow band over which our model is accurate. The in-phase and quadrature-phase components of mixed-down $\psi$ become the state $\mathbf{x}$ used in the Kalman filter, and the in-phase and quadrature-phase components of the data channel become the observation $\mathbf{y}$. The matrices $\mathbf{A}$ and $\mathbf{C}$, describing the state dynamics and the relationship between the state $\mathbf{x}$ and observation $\mathbf{y}$ are derived from the our model in light of the lock-in, band-limiting, and discrete time sampling operations.

2) Over the relevant, narrow band near the resonant peak there is no distinction between viscous and structural damping. 


\section{RESULTS AND DISCUSSION}

\section{A Removing the artifact}

To explore the effectiveness of the Kalman filter in identifying the contribution of the violin modes to the detector output we have applied it to data taken in November 1994 at the LIGO 40M prototype detector. In this instrument the fundamental violin mode resonances are all in the $(571.6,605.425) \mathrm{Hz}$ band.

The upper and lower panels of figure 1 show the power spectra of the interferometer output in a $45 \mathrm{~Hz}$ band between 565.0 and $610.0 \mathrm{~Hz}$ before and after the subtractive removal of the Kalman filter estimate of the violin mode contribution to the detector output. The filter identifies the contribution of the mode to the detector output, allowing us to suppress this artifact by $40 \mathrm{~dB}$. The residual bumps positioned in the wings of the removed lines, are non-linear artifacts: the violin mode amplitudes are so large that they modulate the detector transfer function, up-converting other detector noise frequency-modulating the violin mode signal itself.
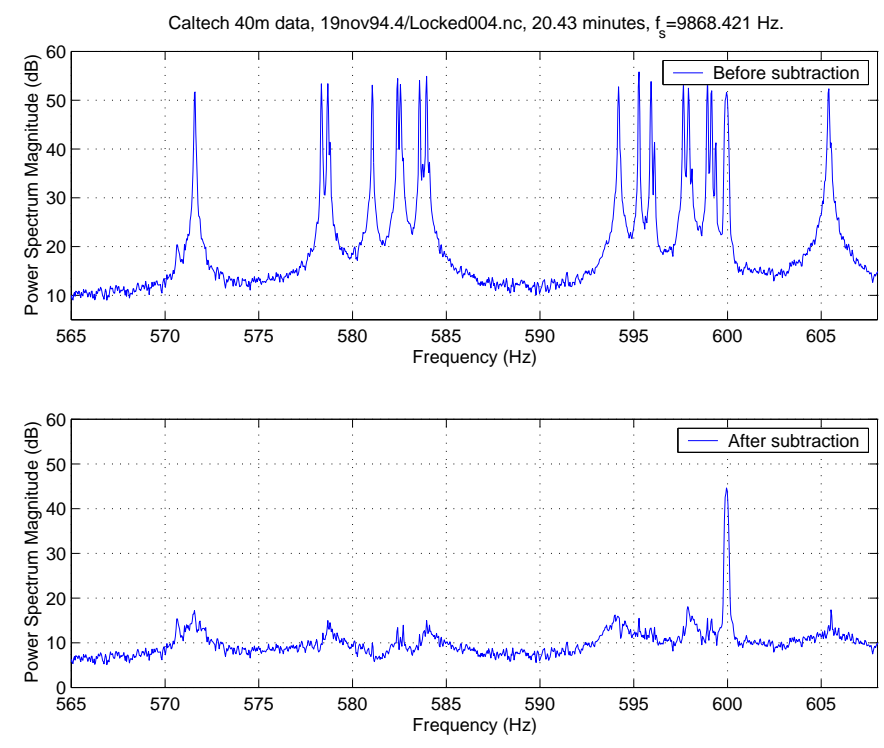

FIGURE 1. Power spectrum of the 40 meter data showing the violin modes between 571 and $605 \mathrm{~Hz}$ before and after subtraction of the Kalman estimates for all the modes. The remaining line feature is the 9th harmonic of the $60 \mathrm{~Hz}$ power main. Features like these are dealt with in other ways $[2-5]$. 


\section{B Statistics of the artifact and residual}

Some simple exploratory statistics show the value of identifying and removing the known instrumental artifacts from the data stream.

Figure 2 shows a histogram of the sample amplitude relative to the RMS sample amplitude for the data channel before (top) and after (middle) removal of the Kalman filter estimate of the violin mode contribution, and (bottom) for the estimated violin mode contribution itself. In an ideal world the measurement noise and the process noise are Normal; correspondingly, each of these distributions should be Rayleigh and appear, in these figures, as straight lines. Departures from a straight line thus imply non-Gaussian noise statistics.

Comparing the three panels in figure 2 shows that the violin mode artifact contributes significantly to the non-Gaussian component of the noise in the detector data channel. Since gravitational waves do not excite the violin modes, this excess noise component is strictly technical. Generalizing to the full-scale detector, removing artifacts like these, together with their associated excess noise, from the data stream before analysis thus strengthens our ability to make significant statements regarding the detection of gravitational waves in the residual.
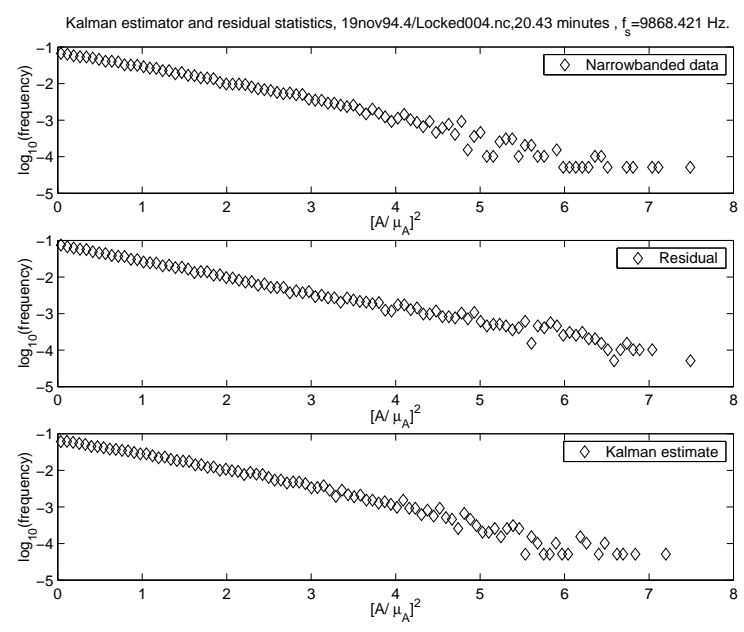

FIGURE 2. Histograms of the square of Kalman estimated amplitude divided by mean amplitude for (top) data in the narrow-band (570-599.5 Hz) having the fundamental violin modes, (middle) residual after subtraction of the Kalman estimates of the modes from the data and (bottom) the Kalman estimated violin modes. Deviations from Gaussianity occur with much lower frequency in the residual than in the mode contribution to the observation.

\section{Violin modes vs. gravitational waves}

The Kalman filter estimates the violin mode contribution to the gravitational wave channel. If that estimate is influenced by the presence of a gravitational wave 
signal then removing the estimated contribution may distort evidence of the wave in the output.

The Kalman filter identifies the violin mode contribution through its dynamics. Since the evolution with time of expected gravitational wave signals is different than the dynamics of the mode contribution to the detector output, we expect that the Kalman estimates of the violin mode contribution will not be influenced by the presence of a gravitational wave signal.

It is useful to consider two different kinds of sources: burst sources, such as inspiraling neutron star binary systems, and periodic sources, such as a pulsar at a frequency near to but not identical with the violin mode frequency.

Nearly all the signal to noise ratio signal-to-noise from an inspiraling binary is contributed when the signal is in the band from about $70 \mathrm{~Hz}$ to $250 \mathrm{~Hz}[6,7]$ : what happens in the band near the violin mode is is inconsequential. Similarly, the filter output is entirely unaffected by what happens in the band where the $\mathrm{S} / \mathrm{N}$ is deposited. We have verified this by forming estimates of the violin mode state from the LIGO 40M prototype data, and from the same data set but with an added, simulated gravitational wave signal corresponding to a coalescing neutron star binary. Even for a very strong signal there is no difference in the predicted mode amplitudes when the signal was in the relevant band.

In separate experiments we have looked at how well the Kalman filter rejects nearby monochromatic signals, such as might arise from a pulsar. As long as the signal is greater than a linewidth away from the violin mode, the estimated state is unaffected by the periodic signal; correspondingly, the periodic signal is unaffected the subtractive removal of the estimated contribution of the mode to the output.

\section{Monitoring the wire state}

The Kalman filter estimates separately the state of each violin mode; correspondingly, we can monitor the state of each of these wires separately. It is convenient to represent the state in terms of its amplitude and phase (as opposed to generalized coordinate and momentum); additionally, it is convenient to express the amplitude as an instantaneous measure of the energy in the mode, expressed in terms of temperature. Excess noise will raise the effective temperature of the mode, while the projection of the corresonding mirror motion normal to the optical axis will lower the effective temperature.

\section{SUMMARY}

Thermal and technical noise that disturbs the violin modes of the substrate suspension moves the mirrors, leading to a strong, narrow band artifact in the gravitational wave channel. Gravitational waves, on the other hand, also change the distance between the mirrors, but without moving or otherwise exciting these modes. The Kalman filter described here distinguishes between excitations due 
to gravitational waves and those due to thermal or other excitations of the violin modes, allowing us to eliminate a broad class of technical noise sources that might otherwise masquerade as gravitational wave bursts.

A Kalman filter uses the known dynamics of the modes to distinguish between the mode "signal" and other contributions to the measured detector output: i.e., it detects the violin modes. This distinguishes it from other methods (e.g., multitaper methods, linear notch filters [8]) which purport to characterize or remove artifacts, but which in fact simply suppress all contributions to the noise within a narrow band, not distinguishing violin mode from other contributions.

The computational cost of identifying and removing the violin modes using the Kalman filter described here is negligible: an interpreted Matlab [9] implementation on a low-end workstation runs at greater than $20 \times$ the detector's real-time sample rate. A compiled implementation, with attention paid to optimization, an additional speed-up of 10 or more can be expected.

We thank Albert Lazzarini for drawing our attention to Kalman Filtering and the LIGO Laboratory for its hospitality during the 1997/8 academic year and for the use

of the 40 meter prototype data. SM thanks S. Mohanty for many valuable insights and LSF thanks P. Fritschel for valuable discussions. This work was supported by NSF awards PHY 98-00111 and 99-96213.

\section{REFERENCES}

1. R. Kalman, Trans. ASME, J. Basic Eng. 82D, (1960).

2. A. Sintes and B. Schutz, Phys. Rev. D 58, 122003 (1998).

3. L. S. Finn et al., Toward gravitational wave detection, 1999, gr-qc/9911001; to appear in the proceedings of the Third Edoardo Amaldi Conference on Gravitational Waves.

4. L. S. Finn and M. F. Huq, Regressing power line artifacts in gravitational wave detector data, 1999, in preparation.

5. B. Allen, W. Hua, and A. Ottewill, Automatic cross-talk removal from multi-channel data, gr-qc/9909083.

6. L. S. Finn, Phys. Rev. D 53, 2878 (1996).

7. L. S. Finn, Technical Report No. T970167, California Institute of Technology, LIGO Laboratory, California Institute of Technology, Pasadena CA 91125 (unpublished).

8. B. Allen, GRASP: a data analysis package for gravitational wave detection, 1998, available from <http://www.lsc-group.phys.uwm.edu/ ballen/grasp-distribution $>$.

9. Matlab, a technical computing environment for high-performance numeric computations in linear algebra, is a product of The MathWorks, Inc. 